\title{
Rapid diagnostic tests versus peripheral smear in malaria: a comparative study
}

\author{
Kulkarni $\mathbf{P}^{1}$, Varna $\mathbf{I}^{2}$ \\ ${ }^{1}$ Dr Padmaja Kulkarni, Associate Professor, Pathology Department, Kodagu Institute of Medical Sciences, Madikeri, \\ Karnataka 571201, ${ }^{2}$ Dr Varna I, Assistant Professor, Pathology Department, Karwar Institute of Medical Sciences, \\ Karwar, M G Road Karwar, Kodibag, Karwar, Karnataka 581301, India
}

Address for Correspondence: Dr Padmaja Kulkarni, Email id: padmaja.kul21@gmail.com

\begin{abstract}
Introduction: Malaria is one of important vector borne disease in India. It can be fatal if not treated promptly. The early diagnosis and treatment of malaria is essential to prevent complications especially in cerebral malaria. Aims: To evaluate the diagnostic accuracy of Rapid Diagnostic tests (RDT) in the diagnosis of malaria. Methods and Material: Blood samples from all clinically suspected cases of malaria were routinely subjected to peripheral smear examination and RDT for the presence of malaria parasite. Statistical analysis used: Sensitivity, Specificity, Positive predictive value and Negative predictive value were analyzed using standard formulae. Results: RDT are having Sensitivity, specificity, Positive Predictive Value and Negative Predictive value of 100\%, 96.7\%, 92.5\% and 100\% respectively. Conclusions: RDTs are equally or more sensitive and specific than peripheral smear. Newer Pf /Pv specific antigen RDT kits can distinguish mixed and PF infections. However further studies are required to assess cost effectiveness and efficiency of different RDTs.
\end{abstract}

Keywords: Malaria diagnosis, Rapid Diagnostic test, Diagnostic accuracy

\section{Introduction}

Malaria is one of the important vector borne disease in India. It can be fatal if it is not diagnosed and treated early. $89 \%$ of India's population is residing in malaria prone region with $22 \%$ in high transmission (> 1 case per 1000 population) areas and $67 \%$ in low transmission (0-1 cases per 1000 population) areas. National Vector Borne Disease Control Programme (NVBDCP) has currently reported $0.7-1.6$ million confirmed cases of malaria leading to 400-1,000 deaths annually [1]. Malaria is caused by five Plasmodium species with different geographic distribution; Plasmodium Falciparum and Plasmodium Vivax are more common in India. Conventional Peripheral smears, Quantitative Buffy Coat, and Rapid Diagnostic tests (RDT) are commonly available diagnostic tests for malaria [2]. Recently RDTs are increasingly used for malaria diagnosis especially in regions where microscopic facilities do not exist. Around 200 different RDT kits having a wide range of specificity and sensitivity are

Manuscript received: $04^{\text {th }}$ December 2016

Reviewed: $10^{\text {th }}$ December 2016

Author Corrected: $18^{\text {th }}$ December 2016

Accepted for Publication: $28^{\text {th }}$ December 2016 commercially available in the market, However, to be useful as a screening test, any diagnostic test should possess $>95 \%$ sensitivity. This study was conducted to evaluate efficiency of Rapid Diagnostic test in comparison to peripheral smear examination for diagnosis of malaria.

\section{Methods and Materials}

This was a prospective study conducted in the department of Pathology at SIMS, Mangalore, Karnataka. Duration of the study was from April 2015 to August 2016.

Sample Collection: During this period, 1835 blood samples were received for malaria diagnosis from clinically suspected cases. Blood samples were collected in EDTA vacutainer tube. Peripheral smears were made on a clean glass slides with a drop of blood, air dried and stained with Leishman stain. Smears were thoroughly examined under oil immersion for the presence of malaria parasite. Of 1835 samples, 600 samples were randomly selected and Rapid Diagnostic 


\section{Research Article}

test was performed using Antigen based Pf (HRP-II) and PV (pLDH) specific kit. Procedure was performed as per manufacturer's instructions. About $5 \mathrm{l} 1$ of blood was put in sample well with the help of disposable loop provided with the kit. 4 drops of assay diluent provided with the kit was added to second well. Results were interpreted after $15 \quad-20$ minutes. Results were interpreted as negative when only control band appeared with two negative test bands and as mixed infection when control band and two test bands appeared. It was interpreted as Plasmodium Vivax infection when PV band appeared along with control band. Plasmodium Falciparum was diagnosed when Pf band and control band appeared.
Inclusion Criteria: Clinically suspected cases of malaria which had both peripheral smear and Rapid diagnostic tests performed on the same blood sample.

Statistical Analysis: Sensitivity, specificity, Positive Predictive value (PPV) and Negative predictive value (NPV) were calculated using standard formulae considering Peripheral smear diagnosis as gold standard. Sensitivity $=\mathrm{TP} / \mathrm{TP}+\mathrm{FN}$, Specificity $=$ $\mathrm{TN} / \mathrm{TN}+\mathrm{FP}, \mathrm{PPV}=\mathrm{TP} / \mathrm{TP}+\mathrm{FP}, \mathrm{NPV}=\mathrm{TN} / \mathrm{TN}+\mathrm{FN}(\mathrm{TP}$ -true positive, $\mathrm{TN}$ - true negative, FP - False Positive, FN - False Negative)

\section{Results}

In the present study six hundred samples were evaluated for the presence of malaria parasite by conventional peripheral smear examination and Rapid Diagnostic Test. Of the 600 Peripheral smears studied, 175 showed positive for malarial parasite. Plasmodium Vivax $(\mathrm{Pv})$ was diagnosed in 173 Cases, Plasmodium Falciparum (Pf) was identified in one case and one smear showed mixed infection with both Plasmodium Vivax and Plasmodium Falciparum.

Rapid Diagnostic test showed 189 positive cases, of which 178 were plasmodium Vivax, four cases were Plasmodium Falciparum and seven cases showed mixed infection with Falciparum and Vivax. Sensitivity, specificity, Positive Predictive Value and Negative Predictive value were 100\%, 96.7\%, 92.5\% and $100 \%$ respectively.

Table-1: Showing comparison of Peripheral smears and Rapid Diagnostic Tests diagnoses

\begin{tabular}{|l|c|c|}
\hline Results & Peripheral smears & Rapid Diagnostic tests \\
\hline Positive cases & $175 / 600(29.1 \%)$ & $189 / 600(31.5 \%)$ \\
\hline Plasmodium Vivax & 173 & 178 \\
\hline Plasmodium Falciparum & 01 & 04 \\
\hline Mixed infection & 01 & 07 \\
\hline Negative & $425 / 600(70.9 \%)$ & $411 / 600(68.5 \%)$ \\
\hline Total cases & 600 & 600 \\
\hline
\end{tabular}

\section{Discussion}

Accurate diagnosis and early treatment of malaria is essential to reduce mortality and morbidity due to malaria. The various modalities to diagnose malaria are conventional peripheral smear, Quantitative Buffy coat, antigen based Rapid diagnostic kits and Molecular studies (PCR). As per 2011 WHO report, the sensitivity of microscopic examination is less than $75 \%$. It is a common practice in many parts of India to treat febrile patients with antimalarial drugs even after negative microscopic examination which has resulted in resistance to commonly used drug chloroquine. Now the concern is emergence of drug resistance to artemisinin therapy if empirical therapy is followed and this may not be cost effective also as artemesisnin is more expensive than chloroquine [3].
There are more than 60 brands of RDTS in the market based on different combination of antigen specificity. Previous studies have shown RDTs that detects Histidine Rich Protein type 2 (HRP-2) are more sensitive in diagnosing Plasmodium falciparum whereas those detecting lactate dehydrogenase (LDH) enzyme are more specific for P.Vivax diagnosis [4].

In the present study RDT with Pf (HRP 2) and PV (pLDH) specificity were used. Past studies have also proven that the cost of malaria treatment can be reduced by $24 \%$ by using RDT and $46 \%$ by microscopy against presumptive treatment [5]. In the present study out of 600 patients $189(31.5 \%)$ were positive and 411 
(68.5\%) were negative to RDT whereas 175 (21.1\%) were positive and $425(70.9 \%)$ were negative on microscopic examination. Similar findings were also reported in a study conducted by Rajini Kurup [6].

Previous studies have shown sensitivity and specificity ranging from 84 to $100 \%$ for RDT $[7,8,9]$. In the present study we found $100 \%$ and $96.7 \%$ respectively. In our study peripheral smear were negative in 14 cases that showed positivity with RDT.

These peripheral smears were retrieved and studied again. In few cases parasite density was very low and occasional parasite was noted after careful screening of the smears and few cases were partially treated cases before visiting this hospital. Compared to Peripheral smear RDTs are more sensitive and specific for diagnosis of P Falciparum and mixed infections.

This is important because Falciparum causes severe disease and has high mortality requiring urgent intervention, whereas P. Vivax needs to be treated with primaquine to prevent relapses of malaria.

The advantages of RDTs are that it is simple, easy to perform, no instruments or electricity required and interpretation is also easy. But the disadvantage is parasite density cannot be assessed and cannot be used to assess response to treatment as it can be positive for 7-14 days after treatment [10].

And with $>60$ brands being marketed in India there is always confusion about which RDT kit to use. Pf /Pan specific RDTs cannot differentiate mixed infection (Pf with $\mathrm{Pv}$ ) from $\mathrm{P}$. Falciparum infections. But recently it is found P.Vivax also can lead to serious disease and no longer can be considered as benign malaria [2].

Hence when Pv/Pan specific RDT kit is used, mixed infections are to be confirmed with peripheral smear examination. However, newer $\mathrm{Pf} / \mathrm{Pv}$ specific RDT kits can differentiate mixed from $\mathrm{P}$. falciparum infections.

Peripheral smear though inexpensive of the two is laborious to perform, less sensitive, requires electricity, microscope and skilled technician to interpret. Results depend on quality of the smears [11].

But the advantages of peripheral smears are it is cheaper than RDT, parasite density can be assessed and it can also be used as quality control measure to check efficiency of RDTs.

\section{Conclusion}

Peripheral smears are considered to be gold standard for diagnosis of malaria. RDTs can be more sensitive and specific than peripheral smears. Newer Pf /Pv specific antigen card can distinguish mixed and PF infections. However further studies are required to assess cost effectiveness and efficiency of different RDTs.

Funding: Nil, Conflict of interest: None initiated, Permission from IRB: Yes

\section{References}

1. WHO. World Malaria Report 2014. WHO, Geneva. 2014. Available at http://apps.who.int/iris/bitstream/10665/144852/2/9789 241564830_eng.pdf

2. Yan J, Nana Li, Xu Wei, Peipei Li, Zhenjun Z, Lili $\mathrm{W}$ et al.: Performance of two rapid diagnostic tests for malaria diagnosis at the China-Myanmar border area. Malaria Journal 2013 12:73.

3. Wilson LM. Laboratory Diagnosis of Malaria conventional and Rapid Diagnostic Methods. Arch Pathol Lab Med. 2013 Jun;137(6):805-11. doi: 10.5858/arpa.2011-0602-RA.

4. Chansuda W, Mazie BJ, Muth S, Awalludin S, Walther HW. A Review of Malaria Diagnostic Tools: Microscopy and Rapid Diagnostic Test(RDT). Am J Trop Med Hyg. 2007 Dec;77(6 Suppl):119-27.

5. Osei-Kwakye K, Asante KP, Mahama E, Apanga S, Owusu R, et al. (2013) The Benefits or Otherwise of Managing Malaria Cases with or without Laboratory Diagnosis: The Experience in a District Hospital in Ghana. PLoS ONE 8(3): e58107. doi:10.1371/journal.pone.0058107

6. Rajini Kurup, Rena Marks. A comparison of microscopic examination and rapid diagnostic tests used in Guyana to diagnose malaria. Malaria Reports 2012; 2:e2.doi:10.4081/malaria.2012.e2

7. Hada S, Das ML, Singh YL. Diagnostic methods of malaria in Eastern Nepal: a comparative study of traditional and two rapid diagnostic tests. Nepal Med Coll J 2011; 13(4): 261-266.

8. Ouattara A, Doumbo S, Saye R, Beavogui AH, Traoré B, Djimdé A, Niangaly A, Kayentao K, Diallo M, Doumbo OK, Thera MA. Use of a pLDH-based 


\section{Research Article}

dipstick in the diagnostic and therapeutic follow-up of malaria patients in Mali. Malar J. 2011 Nov 24;10:345. doi: 10.1186/1475-2875-10-345.

9. Schrot-Sanyan S, Pagnier SG, Bacar AA, Sirima SB, Candolfi E. Malaria relevance and diagnosis in febrile Burkina Faso travellers: a prospective study. Malar J. 2013 Aug 2;12:270. doi: 10.1186/1475-2875-12-270.
10. Mendiratta DK, Bhutada K, Narang R, Narang P. Evaluation Of Different Methods For Diagnosis Of P. Falciparum Malaria. Indian J Med Microbiol. 2006 Jan;24(1):49-51.

11. Salmani MP, Mindolli PB, Peerapur BV. Comparative Study of Peripheral Blood Smear, QBC and Antigen Detection in Malaria Diagnosis. Journal of Clinical and Diagnostic Research 2011;5(5): 967-969.

\section{How to cite this article?}

Kulkarni P, Varna I. Rapid diagnostic tests versus peripheral smear in malaria: a comparative study . Trop J Path Micro 2016;2(3):179-182.doi: 10.17511/jopm.2016.i03.17 